\title{
ESTUDIO CUALI-CUANTITATIVO SOBRE LA ATENCIÓN DE SALUD DE NIÑOS MENORES DE TRES AÑOS EN ESTABLECIMIENTOS DE SALUD DE NUEVE REGIONES POBRES DEL PERÚ
}

\author{
Luis Germán Cordero Muñoz 1,2,a, Cecilia Montes Jave1,3,b, José Enrique Velásquez Hurtado ${ }^{1,2, a}$, \\ Yuleika Rodríguez Calviño ${ }^{1, a}$, Walter Eduardo Vigo Valdez ${ }^{1, c}$, Ángel Martín Rosas-Aguirre²,d
}

\begin{abstract}
RESUMEN
Objetivos. Evaluar cinco elementos importantes de la calidad de atención (espacio físico, equipamiento, personal, tiempo para la atención, y consejería) en servicios de crecimiento y desarrollo (CRED) de 18 establecimientos de salud (EE. SS.) en nueve regiones con altos índices de pobreza en Perú. Materiales y métodos. Estudio cuali-cuantitativo realizado en Amazonas, Apurímac, Ayacucho, Cajamarca, Cusco, Huánuco, Huancavelica, Puno y Ucayali, que incluyó la observación directa (OD) durante la atención de CRED, grupos focales (GF) con usuarios (uno por establecimiento de salud), y entrevistas en profundidad (EEP) con trabajadores de salud (dos por establecimiento de salud). Resultados. Se realizaron 18 OD para la verificación de ambientes y equipos, 23 OD de turnos completos de atención, 67 acompañamientos a usuarios de los servicios de CRED, 18 GF (118 madres participantes), y 36 EEP. Las madres y los profesionales de salud coincidieron en que existen limitaciones de infraestructura, equipamiento, materiales y personal que afectarían una atención de CRED de calidad. La demora en la atención, el incumplimiento de horarios, y la falta de calidez en la atención son los aspectos que generaron mayor insatisfacción en los usuarios. Solo en $24,3 \%$ del tiempo total de los turnos observados se realizaron actividades que generaron beneficio al usuario. Por su parte, la consejería estaría cumpliendo el objetivo de promover el mejoramiento de las prácticas de cuidado del niño. Conclusiones. El estudio ha permitido identificar debilidades y áreas de oportunidad para reorientar los servicios de CRED, en el marco de la política de fortalecimiento del primer nivel de atención del sector Salud.
\end{abstract}

Palabras clave: Crecimiento y desarrollo; Cuidado del niño; Calidad de la atención de salud; Investigación cualitativa; prevención \& control (fuente: DeCS BIREME).

\section{QUALI-QUANTITATIVE STUDY ON HEALTHCARE FOR CHILDREN BELOW THE AGE OF 3 AT HEALTH ESTABLISHMENTS IN NINE UNDERDEVELOPED REGIONS OF PERU}

\begin{abstract}
Objectives. To assess five elements of healthcare quality (physical space, equipment, personnel, waiting time, and counseling) in growth and development services (GDS) at 18 healthcare establishments in nine regions of Peru with high rates of poverty. Materials and methods. A quali-quantitative study was carried out in the Amazonas, Apurímac, Ayacucho, Cajamarca, Cusco, Huánuco, Huancavelica, Puno, and Ucayali regions, that included direct observation (DO) during medical assistance in GDS, focus groups (FG, one user per healthcare establishment), and in-depth interviews (IDI) with healthcare workers (two per healthcare establishment). Results. There were $18 \mathrm{DOs}$ carried out to assess environment and equipment, 23 DOs of complete service shifts, 67 accompaniments to users during medical assistance in GDS, 18 FGs (118 participating mothers), and 36 IDIs. Both mothers and healthcare professionals agreed that there were limitations in infrastructure, equipment, materials, and personnel that impacted quality medical assistance in GDS. Delays in medical assistance, failure to comply with schedules, and lack of friendliness when providing medical care were the aspects that produced the greatest user dissatisfaction. Only $24.3 \%$ of the total amount of time spent at the healthcare establishment corresponded to beneficial activities for the user, although the Health Department was meeting the objective to promote improvement of childcare practices. Conclusions. The study was useful in identifying weaknesses and strengths that may help redirect GDS efforts within the framework of policies aimed at strengthening first-class medical assistance.
\end{abstract}

Key words: Growth and development; Child care; Quality of health care; Qualitative research; prevention \& control (source: MeSH NLM).

\footnotetext{
Programa de Apoyo a la Reforma del Sector Salud (PARSALUD II). Lima, Perú.

Universidad Peruana Cayetano Heredia. Lima, Perú.

Asociación "Incluir sin Fronteras". Lima, Perú.

Médico cirujano, magister en Salud Pública; ${ }^{\mathrm{b}}$ licenciada en Nutrición, magíster en Epidemiología; ${ }^{\mathrm{c}}$ médico cirujano, magíster en Gerencia de Proyectos y Programas Sociales; ${ }^{\circledR}$ médico cirujano, magíster en Salud Pública, doctor en Salud Pública

Recibido: 22-05-15 Aprobado: 21-10-15
}

Citar como: Cordero-Muñoz LG, Montes-Jave C, Velásquez-Hurtado JE, Rodríguez-Calviño Y, Eduardo Vigo-Valdez W, Rosas-Aguirre AM. Estudio cualicuantitativo sobre la atención de salud de niños menores de tres años en establecimientos de salud de nueve regiones pobres del Perú. Rev Peru Med Exp Salud Publica. 2016;33(1):21-31. doi: 10.17843/rpmesp.2016.331.1922 


\section{INTRODUCCIÓN}

No obstante la disminución de la pobreza en los últimos años ${ }^{(1)}$, la prevalencia de desnutrición crónica infantil en el Perú continúa alta, afectando en promedio al 13,1\% de los niños menores de 5 años ${ }^{(2)}$, pero con cifras que llegan al $25,3 \%$ en zonas rurales. Los efectos de la desnutrición en la infancia pueden ser devastadores y duraderos, impactando el desarrollo integral (físico, cognitivo, emocional) del niño, así como su rendimiento escolar, y su futura productividad en el trabajo ${ }^{(3-5)}$.

El desarrollo integral en la infancia es fundamental para la construcción del capital humano y social, elemento importante para romper el ciclo vicioso e intergeneracional de la pobreza y reducir las brechas de inequidad $(6,7)$. El desarrollo infantil es definido como los cambios en las estructuras físicas y neurológicas, cognitivas y de comportamiento, que emergen de manera ordenada y son relativamente duraderos. Es un proceso que comienza desde la gestación, y que envuelve varios aspectos que van desde el crecimiento físico, pasando por la maduración neurológica, motora, comportamental, cognitiva, social y afectiva del niño ${ }^{(7)}$.

La vigilancia del crecimiento y desarrollo infantil comprende todas las actividades relacionadas con la promoción del desarrollo normal y a la detección de los problemas en el desarrollo ${ }^{(8)}$. La identificación de riesgos biológicos (como el crecimiento intrauterino limitado, la desnutrición crónica y/o las enfermedades infecciosas agudas y crónicas) y/o a riesgos psicosociales (como la falta de oportunidades de aprendizaje y/o la baja calidad de la interacción entre el niño y sus cuidadores) en la población infantil ofrece la oportunidad de intervenir precozmente y evitar graves consecuencias en su desarrollo (9). Pero para que sean efectivas, tanto la vigilancia como las intervenciones, deben alcanzar la mayor cobertura de la población objetivo infantil y sus actividades deben realizarse siempre asegurando la calidad de las mismas.

De acuerdo con la Norma Técnica del Ministerio de Salud del Perú (MINSA), el seguimiento se desarrolla durante los controles de crecimiento y desarrollo (CRED) de los niños menores de 5 años. Se debe realizar un conjunto de actividades periódicas y sistemáticas con el objetivo de vigilar de manera adecuada y oportuna el CRED del niño; detectar de manera precoz y oportuna riesgos, alteraciones o trastornos, así como la presencia de enfermedades, facilitando su diagnóstico e intervención oportuna disminuyendo deficiencias y discapacidades ${ }^{(10)}$.

Durante el control CRED, el profesional de salud utiliza la entrevista, la observación, el examen clínico, anamnesis, exámenes de laboratorio, aplicación de instrumentos de evaluación del desarrollo e instrumentos para valoración del CRED del niño ${ }^{(10)}$. Además, brinda consejería a los padres con el propósito de incentivar y fortalecer las prácticas apropiadas, y corregir aquellas de riesgo.

En este contexto, asegurar la calidad de las actividades y procedimientos que se realizan en los controles de CRED es esencial para el bienestar y desarrollo de los niños. Según Donabedian, la calidad debe ser entendida como el logro de los mayores beneficios posibles de la atención médica con los menores riesgos para el paciente, y su evaluación incluye el análisis de tres dimensiones: estructura (comodidades, instalaciones físicas, equipamiento, organización), procesos (uso eficiente de los recursos humanos y técnico-científicos, procedimientos y acciones realizadas en la consulta y en los trámites) y resultados (cambios en el estado de salud y la percepción general de la atención recibida o grado de satisfacción) ${ }^{(11)}$.

El monitoreo de la calidad de los servicios de CRED está enmarcado dentro las líneas de investigación operativa del MINSA que buscan evaluar la efectividad del programa de CRED en la mejora de la salud, nutrición y calidad de vida de los niños ${ }^{(10)}$, así como identificar factores que facilitan o limitan esta. En este contexto, se diseñó un estudio cuali-cuantitativo que evaluó cinco elementos relacionados con la calidad de atención (espacio físico y equipamiento en la dimensión estructura; y personal, tiempo para la atención, y consejería de la dimensión procesos) en los servicios de CRED de nueve regiones con altos índices de pobreza en el Perú.

\section{MATERIALES Y MÉTODOS}

Estudio cuali-cuantitativo exploratorio-descriptivo realizado entre diciembre 2012 y marzo 2013 en el ámbito de intervención del Programa de Apoyo a la Reforma del Sector Salud II (PARSALUD II) ${ }^{(12)}$, que incluye zonas rurales de 202 distritos (316 451 hogares según censo nacional ${ }^{(13)}$ ) de las nueve regiones con los más altos índices de pobreza del país (1): Amazonas, Apurímac, Ayacucho, Cajamarca, Cusco, Huánuco, Huancavelica, Puno y Ucayali. Se seleccionaron aleatoriamente dos establecimientos de salud (EE. SS.) por región (Tabla 1), en base al marco muestral de 94 EE. SS., priorizados por el PARSALUD II para la realización de obras de infraestructura y/o equipamiento de servicios. Dos poblaciones fueron seleccionadas para el estudio: madres de niños menores de 36 meses que acudieron a EE. SS. para los controles de CRED de sus niños, y profesionales de salud de los EE. SS. (jefes de EE. SS. y responsables de servicios de CRED).

Se usaron tres técnicas cualitativas para la recolección de datos: observación directa (OD), grupos focales (GF) y entrevistas en profundidad (EEP) ${ }^{(14)}$. La OD fue utilizada con cuatro propósitos. Primero, para determinar si el 
Tabla 1. Establecimientos de salud incluidos en el estudio y ambientes y equipamiento de los servicios de crecimiento y desarrollo

\begin{tabular}{|c|c|c|c|c|c|}
\hline \multirow{2}{*}{\multicolumn{2}{|c|}{ Establecimientos de salud }} & \multirow{2}{*}{ Región } & \multirow{2}{*}{$\begin{array}{c}\begin{array}{c}\text { Ambiente } \\
\text { exclusivo }\end{array} \\
\text { Sí/No }\end{array}$} & \multicolumn{2}{|c|}{ Equipos mínimos para medir peso y estatura } \\
\hline & & & & Menores ( $\leq 1$ año) & Mayores (2-5 años) \\
\hline $\mathrm{CS}$ & Lonya Grande & Amazonas & No & Sí & No \\
\hline $\mathrm{HA}$ & Santiago Apóstol & Amazonas & No & No & Sí \\
\hline CS & Haquira & Apurímac & No & No & No \\
\hline $\mathrm{H}$ & Tambobamba & Apurímac & No & No & No \\
\hline CS & Putacca & Ayacucho & Sí & Sí & Sí \\
\hline CS & Vilcashuamán & Ayacucho & No & No & No \\
\hline CS & San Marcos & Cajamarca & No & Sí & Sí \\
\hline $\mathrm{H}$ & San Javier Bellavista & Cajamarca & Sí & No & Sí \\
\hline CS & Paucartambo & Cusco & No & No & No \\
\hline $\mathrm{H}$ & Santo Tomás & Cusco & Sí & No & No \\
\hline CS & Huando & Huancavelica & Sí & Sí & Sí \\
\hline CS & Paucarbamba & Huancavelica & Sí & Sí & Sí \\
\hline CS & Puerto Inca & Huánuco & Sí & No & Sí \\
\hline PS & Margos & Huánuco & Sí & No & No \\
\hline CS & Isivilla & Puno & Sí & Sí & Sí \\
\hline CS & San Anton & Puno & Sí & No & Sí \\
\hline CS & Aguaytia & Ucayali & No & No & No \\
\hline CS & San Fernando & Ucayali & Sí & No & No \\
\hline
\end{tabular}

${ }^{*} \mathrm{CS}$ : centro de salud, HA: hospital de apoyo, $\mathrm{H}$ : hospital.

ambiente del servicio de CRED era exclusivo o compartido con otro servicio, registrar las dimensiones del mismo y verificar la disponibilidad de equipos claves para la evaluación antropométrica (balanzas, infantómetros y tallímetros). Segundo, para observar turnos completos de atención de los servicios de CRED y determinar qué porcentajes del tiempo total del turno son empleados en actividades que agregan valor o beneficio directo al usuario (actividades de diagnóstico, resultados y/o interpretación, consejería, vacunación, y mediciones antropométricas), y en actividades que no benefician directamente (registro de información, administrativo obligatorio, desplazamiento, demoras, personal fuera de servicio durante la atención, etc.). Se procuró realizar al menos una OD de un turno completo por establecimiento de salud. Tercero, para el acompañamiento a usuarios del servicio CRED -previo consentimiento informado firmado- desde su llegada hasta su retiro del establecimiento de salud. Se procuró realizar al menos tres acompañamientos por establecimiento de salud. En cada acompañamiento, se registró el contacto y permanencia (tiempo cronometrado) del usuario con cada ambiente del establecimiento de salud -dentro y fuera del servicio de CRED. Esta información fue posteriormente consolidada gráficamente mediante un croquis con todos los desplazamientos y contactos realizados por los usuarios (diagrama de hilos). Cuarto, para verificar los mensajes de consejería brindados durante las consultas mediante una lista de chequeo. Los mensajes claves verificados estuvieron relacionados con la lactancia materna exclusiva, el lavado de manos, la consejería nutricional, y la administración de suplementos de fierro y vitaminas.
En cada GF participaron 8-10 madres, quienes habían acudido al servicio de CRED para la atención de sus niños días previos a la recolección de información del equipo de investigación en el establecimiento de salud. Se realizó un GF por cada establecimiento de salud. Previo consentimiento informado firmado, utilizando una guía semiestructurada; personal con experiencia en estudios cualitativos realizaron preguntas abiertas para conocer las percepciones de las madres sobre cinco elementos de la atención: a) ambiente y espacio físico del servicio de CRED, b) equipamiento de los servicios, c) disponibilidad de personal adecuado para brindar la atención, d) tiempo en el establecimiento de salud para la atención, y e) mensajes y metodología usada para proporcionar información durante la atención de CRED (lactancia materna, lavado de manos, nutrición, toma de suplementos y vitaminas, signos de alarma). Las EEP fueron realizadas a jefes de establecimiento de salud y personal del servicio de CRED, previo consentimiento informado. Utilizando una guía semiestructurada, se les realizó preguntas abiertas para conocer sus percepciones sobre los mismos cinco elementos de la atención mencionados arriba. Adicionalmente, durante las EEP a los jefes de los EE. SS. se solicitó la información completa de los turnos y días programados y trabajados por el personal del servicio de CRED. Tanto las guías para GF como para las EEP fueron elaboradas por el equipo de investigación, revisadas por profesionales con experiencia en la gestión y atención de los servicios de CRED del nivel nacional, y finalmente ajustadas luego de su validación en un piloto realizado 
en poblaciones objetivo similares a la del estudio (madres de niños atendidos en servicio de CRED para los GF y profesionales encargados de la atención para las EEP). Todas las sesiones de GF y EEP en el estudio fueron grabadas digitalmente.

Los datos cuantitativos analizados en el estudio fueron: el tiempo cronometrado de permanencia en los EE. SS., los datos recogidos sobre la disponibilidad de profesionales y cantidad de horas de atención en los EE. SS., y los datos recolectados usando la lista de chequeo para verificar los mensajes de consejería brindados durante la atención de CRED. Usando el paquete estadístico $R$ v.3.1.1 se calcularon los tiempos promedio de permanencia en los EE. SS., y la distribución de este en: tiempo de permanencia específicamente en el servicio de CRED, tiempo de permanencia en otro servicio, y tiempo en espera para la atención (tiempo de demora en la atención). De manera similar, se utilizó métodos de estadística descriptiva para mostrar los resultados de la distribución del tiempo en actividades que agregan o no valor durante la observación de turnos completos de atención de CRED, para estimar la frecuencia relativa de cumplimiento de las horas programadas en un mes para la atención de CRED por establecimiento de salud, y para presentar las características de la consejería brindada.

Previo al análisis de datos cualitativos, se realizó la transcripción completa de las grabaciones de GF y EEP. El análisis estuvo a cargo de dos investigadores con experiencia en análisis cualitativo, quienes leyeron cuatro transcripciones (dos de selva y dos de sierra) para la identificación de temas de la lectura y posterior desarrollo de los libros de códigos iniciales. Luego, los mismos investigadores procedieron a codificar las transcripciones utilizando el software ATLAS.ti v6.0. (Scientific Software Development $\mathrm{GmbH}$, USA), compararon línea a línea la codificación y luego de llegar a un consenso, finalizaron los libros de códigos. Con la disponibilidad de dichos códigos se procedió a codificar las demás transcripciones. Se realizó un análisis cualitativo de contenido y de síntesis orientado a identificar tanto las percepciones maternas como de los profesionales sobre los cinco elementos de la atención antes mencionados, y los resultados fueron triangulados entre sí y con los datos obtenidos durante la observación.

Se obtuvieron los permisos correspondientes de las autoridades de salud. Previo al inicio de la OD de turnos de atención, acompañamientos a usuarios, GF y EEP, se obtuvieron los consentimientos informados correspondientes. El estudio tuvo aprobación del Comité de Ética de la Organización No Gubernamental Asociación Benéfica Prisma, Lima, Perú (CE0047.13).

\section{RESULTADOS}

Se realizaron 18 OD de los ambientes y equipos de los servicios de CRED en los EE. SS., 23 OD de turnos completos de atención, un total 67 acompañamientos a madres y sus niños durante toda su permanencia en los EE. SS., 18 GF (118 madres participantes), y 36 EEP a trabajadores de salud.

\section{AMBIENTE, ESPACIO FÍSICO Y EQUIPAMIENTO DE LOS SERVICIOS}

En diez EE. SS. se encontraron servicios de CRED con ambientes exclusivos; en los otros ocho EE. SS. Ios ambientes eran compartidos con un segundo servicio CRED, o con inmunizaciones y estimulación temprana (Tabla 1). Del total de 15 ambientes con dimensiones disponibles, solo uno estaba por debajo de los $12 \mathrm{~m}^{2}$ de área. Respecto al equipamiento operativo mínimo para la evaluación antropométrica de los niños, seis EE. SS. contaban con balanza de mesa e infantómetro para pesar y tallar a niños con edad $\leq 1$ año, y nueve EE. SS. con balanza de pie y tallímetro (o balanza metálica con tallímetro) para pesar y tallar niños de 2 a 5 años de edad. En los GF se evidenciaron percepciones negativas de las madres sobre los ambientes de los servicios de CRED, sus salas de espera, y su equipamiento (Tabla 2). Según ellas, en varios de los EE. SS. los espacios de los servicios

Tabla 2. Percepciones sobre los ambientes y equipamiento desde la perspectiva de los usuarios y profesionales de salud

\begin{tabular}{|c|c|}
\hline Usuarios & Profesionales de salud \\
\hline $\begin{array}{l}\text { Ambientes reducidos } \\
\text { "...el lugar es muy reducido, es pequeño, en donde al costadito está el } \\
\text { tallímetro, la balanza está a un costado, yo creo que debe ser un poco } \\
\text { más amplio y el lugar también de que hay muchos pacientes que pasan } \\
\text { por la puerta de los niños, donde hay enfermos que pasan hay todo } \\
\text { personas, entonces debe ser un lugar más adecuado" (GF4 Apurímac). }\end{array}$ & $\begin{array}{l}\text { Ambientes reducidos y antiguos } \\
\text { "En los consultorios, del área del niño por ejemplo, como son ambientes } \\
\text { antiguos como yo lo había referido que tienen como más de cuarenta años } \\
\text { de construcción (...) no han hecho el mantenimiento (...) son hacinados, } \\
\text { son pequeños, donde se llenan los instrumentos, por ejemplo los tallímetros, } \\
\text { todo ello, actualmente de acuerdo a las normas, los ambientes debe ser más } \\
\text { apropiados para brindar una atención más cómoda..." (EEP Ayacucho) }\end{array}$ \\
\hline \multicolumn{2}{|l|}{$\begin{array}{l}\text { Localización y disposición de ambientes para consulta y espera Dis } \\
\text { inapropiados } \\
\text { "Que hayga [sic] un lugar donde los niños estén tranquilos, por que } \\
\text { ejemplo donde ellos pueden jugar, mientras esperamos la cita, la hora la s } \\
\text { que nos van a llamar, eso por ejemplo y otro sería de que no pasen ha } \\
\text { otros pacientes que estén en tratamiento por ejemplo, al ladito del hec } \\
\text { laboratorio, que todo el mundo viene a hacer la muestra ahí, entonces } \\
\text { luego puede haber contagio a niños" (GF4 Apurímac). }\end{array}$} \\
\hline $\begin{array}{l}\text { Equipamiento limitado } \\
\text { "Su balanza, a veces esta malograda esa balanza, a veces pesa } \\
\text { menos, a veces pesa más" (GF13 Huanuco). } \\
\text { "Hay una balanza ahí que se supone tiene que pesar a los bebes, ¿no es } \\
\text { cierto? hay unas balancitas como camitas que son más cómodas. (Aquí } \\
\text { tenemos) una balanza con una bolsa que de pronto se puede caer, le duele } \\
\text { la espaldita, yo tengo miedo poner en esa bolsa para que se pese. Y no hay } \\
\text { una camita donde reposarle para poder cambiarle su ropita" (GF17 Ucayali). }\end{array}$ & $\begin{array}{l}\text { Equipamiento y materiales limitados } \\
\text { "...balanzas, esa de allí, por ejemplo, tiene una gran diferencia con la } \\
\text { balanza de Triaje. ...La diferencia es por } 200,300 \mathrm{~g} \text { y eso hace mucho... } \\
\text { (EEP Cusco). } \\
\text { i "...en CRED, si te digo que no tengo ambiente no tengo ni un escritorio, } \\
\text { no hay nada para un paciente... [Material] de estimulación, nos falta. No } \\
\text { / contamos con videos" (EEP Puno). }\end{array}$ \\
\hline
\end{tabular}


de CRED son reducidos para la atención y localizados en zonas no seguras (ej. segundo piso o cerca de laboratorio). Además, fue frecuentemente referido que los servicios carecen de equipos y materiales para la evaluación de CRED. Por su parte, en las EEP los profesionales de la salud manifestaron que la infraestructura y las dimensiones de los ambientes destinados al servicio de CRED en varios de los EE. SS. no llegan a cumplir los estándares requeridos, y los equipos antropométricos y materiales disponibles son muy antiguos y/o se encuentran deteriorados.

\section{DISPONIBILIDAD DE PERSONAL PARA LAATENCIÓN}

La mayoría (13) de EE. SS. programaron dos turnos diarios para el servicio de CRED que duraban entre 5 a $6 \mathrm{~h}$ (Tabla 3). En el mes de enero de 2013, entre los 14 EE. SS. con información disponible se programaron 429 días-persona para la atención del servicio de CRED, de los cuales 348 (81,1\%) fueron efectivamente trabajados. En distintos GF, las madres refirieron que existen insuficientes profesionales para atender a los niños, y cuando están disponibles dichos profesionales no cumplen el horario de atención (Tabla 4). Además, mencionaron la importancia de contar con profesionales con experiencia, pero también que sean capaces de brindar una atención con calidad y calidez. La escasez de personal es un aspecto que también motivó el comentario de los mismos profesionales en las EEP, refiriendo no tener la capacidad para dar atención a todos los usuarios y que ello genera retrasos en la atención de los niños.

\section{TIEMPO EN EL ESTABLECIMIENTO DE SALUD PARA LA ATENCIÓN}

El tiempo promedio de permanencia en los EE. SS. durante el acompañamiento de los usuarios de los servicios de CRED fue 106 minutos (rango: 49-217 minutos), del cual $69,7 \%$ correspondió al tiempo de espera sin recibir atención, $22,4 \%$ al tiempo efectivo en el mismo servicio de CRED y solo 7,9\% al tiempo en otro servicio (Admisión, Caja, Triaje, Laboratorio, Farmacia, etc.) (Tabla 5). Fue común observar que los usuarios debían primero acudir a uno, dos o más servicios (Admisión, Caja, Triaje y/o Estadística) antes de su atención de CRED. Además, la distribución física de los ambientes hizo que en algunos casos los usuarios tuviesen que transitar por los mismos pasadizos en más de una ocasión, regresar a un servicio en más de una ocasión (CRED, Farmacia o Triaje), o tener que subir escaleras con los niños.

Tabla 3. Disponibilidad de profesionales y cumplimiento de horas programadas para la atención de CRED por establecimiento de salud en el mes de enero del 2013

\begin{tabular}{|c|c|c|c|c|c|}
\hline \multicolumn{2}{|c|}{ Establecimiento de salud } & \multirow{2}{*}{$\begin{array}{c}\begin{array}{c}\text { Profesionales en el } \\
\text { servicio }\end{array} \\
\mathbf{n}\end{array}$} & \multirow{2}{*}{$\begin{array}{c}\begin{array}{c}\text { Días-personas } \\
\text { programadas mes }\end{array} \\
\mathbf{n}\end{array}$} & \multicolumn{2}{|c|}{$\begin{array}{l}\text { Días-personas } \\
\text { trabajadas mes }\end{array}$} \\
\hline & & & & $\mathbf{n}$ & $\%$ \\
\hline CS & Lonya Grande & 2 & 26 & 25 & 96,2 \\
\hline HA & Santiago Apóstol & 3 & 26 & NR & 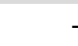 \\
\hline CS & Haquira & 5 & 91 & 57 & 62,6 \\
\hline $\mathrm{H}$ & Tambobamba & 6 & 13 & 13 & 100 \\
\hline CS & Putacca & 3 & 21 & 21 & 100 \\
\hline CS & Vilcashuamán & 6 & 17 & 17 & 100 \\
\hline CS & San Marcos & 4 & 36 & 22 & 61,1 \\
\hline $\mathrm{H}$ & San Javier Bellavista & 2 & 53 & 48 & 90,6 \\
\hline CS & Paucartambo & 5 & 193 & NR & - \\
\hline $\mathrm{H}$ & Santo Tomás & 3 & NR & NR & - \\
\hline CS & Huando & 2 & 46 & 47 & 102,2 \\
\hline CS & Paucarbamba & 2 & 20 & 13 & 65,0 \\
\hline CS & Puerto Inca & 2 & 17 & 16 & 94,1 \\
\hline PS & Margos & 3 & 25 & 22 & 88,0 \\
\hline CS & Isivilla & 1 & 20 & 15 & 75,0 \\
\hline CS & San Antón & 4 & 19 & 14 & 73,7 \\
\hline CS & Aguaytia & 2 & 48 & NR & - \\
\hline CS & San Fernando & 1 & 25 & 18 & 72,0 \\
\hline
\end{tabular}

${ }^{*} \mathrm{CS}$ : centro de salud, HA: hospital de apoyo, H: hospital. NR: no registrado 
Tabla 4. Percepciones sobre la disponibilidad de personal y el tiempo de permanencia en el establecimiento de salud para la atención de CRED desde la perspectiva de los usuarios y profesionales de salud

\begin{tabular}{|c|c|}
\hline Usuarios & Profesionales de salud \\
\hline Insuficiente personal & Insuficiente personal en relación a la demanda de atención \\
\hline $\begin{array}{l}\text { "Hay una sola persona, rapidito le pesa, rapidito le mide su } \\
\text { cabecita, su estatura, todo es rápido porque están amontonados } \\
\text { ahí porque no hay quien le ayude. Una debería estar midiendo, } \\
\text { pesando y la otra escribiendo. Tanto es la cabecita, tanto es el } \\
\text { peso, entonces la otra va escribiendo. No hay quien ayude ahi" } \\
\text { (GF17 Ucayali). }\end{array}$ & $\begin{array}{l}\text { "Ahora como, en este mes se reduce personal por falta de } \\
\text { presupuesto. Ahorita solamente están funcionando dos } \\
\text { consultorios. Dos consultorios y ahí estamos dos los primeros } \\
\text { permanentes. A veces quedamos una sola, y una sale a hacer } \\
\text { trabajo de campo" (EEP Ucayali). }\end{array}$ \\
\hline $\begin{array}{l}\text { Incumplimiento de horario de atención } \\
\text { "Dice cuando no está las doctoras aquí, tienen que esperar, } \\
\text { hasta la hora que lleguen a veces dice de medio atender, se van } \\
\text { y no vuelven y ellas esperan hasta las tres, está diciendo" (GF4 } \\
\text { Apurímac). }\end{array}$ & $\begin{array}{l}\text { “...hoy en día nosotros somos cuatro enfermeros en la } \\
\text { microrred, atendemos la parte asistencial y preventiva, } \\
\text { definitivamente no es suficiente con cuatro enfermeros (...) } \\
\text { ahora hacemos guardias y los cuatro equipos a veces no nos } \\
\text { basta para hacer actividades extramurales, que realmente nos } \\
\text { pide la red..." (EEP Huancavelica). }\end{array}$ \\
\hline
\end{tabular}

\section{Personal sin experiencia}

“...Y faltan doctores profesionales. Puro practicante nomás hay acá" (GF13 Huánuco).

\section{Falta de calidez e indiferencia en la atención \\ “...a veces están como enojadas, no hacen todo lo que deben de hacer, lo pesan, lo tallan 'ya señora, ya', nada más" (GF7 Cajamarca). \\ “...como tengo solamente un bebe, primera vez uno no sabe, yo pensaba que respiraba así, era normal pero yo pensaba que tenía respiración rápida, qué sé yo, los llamaba a cada rato y se molestaban porque los llamaba a cada rato, ni siquiera me explicaban, (¿quién se molestaba?) Las que estaban de turno, las enfermeras. Hasta miedo te daba" (GF8 Cajamarca)}

\section{Percepción negativa sobre la demora en la atención}

"Hay veces que hacen esperar, a veces están sentados, hasta que desespera ya, me canso de estar esperando, me voy, le digo me van a atender o no ... A veces los niños aburrido están, lloran, entonces de preferencia debe haber pa' los niños, pa' los niños eso quisiéramos que las obstetras nos atiendan más rápido" (GF13 Huánuco). “...acá usted ya ha visto, hay poco personal y bastante demanda y no nos abastecemos, y por ejemplo hoy día y cómo le decimos a las madres regrésense, si algunas son de lejos y otras que no, que nos atiendan (exigen)..." (EEP Cajamarca).

"Yo a veces me siento aburrida... porque vengo yo... mayormente vengo yo mayormente en la tarde, vengo a partir de las 2:30 y me voy a mi casa a las 6:00 o 7:00 de la noche, mucho demora... no sé" (GF7 Cajamarca).

"Porque te hacen perder... Porque tenemos... para estar aquí, tenemos que esperar horas y horas... Después casi no te atienden y al otro lado igual ahí, también te hacen esperar. No sé dónde se van las enfermeras, los que atienden aquí, después nos pasan la enfermería y, se demoran, se conversa por teléfono" (GF5 Ayacucho). 
Tabla 5. Distribución del tiempo de permanencia en el establecimiento de salud

\begin{tabular}{|c|c|c|c|c|c|c|}
\hline \multirow{2}{*}{\multicolumn{2}{|c|}{ Establecimiento de salud }} & \multirow{3}{*}{$\begin{array}{c}\text { Número de } \\
\text { mediciones } \\
\text { realizadas }\end{array}$} & \multirow{3}{*}{$\begin{array}{c}\text { Tiempo } \\
\text { promedio en } \\
\text { EE. SS. } \\
\text { Minutos } \\
\end{array}$} & \multicolumn{3}{|c|}{ Distribución del tiempo de permanencia en el EE. SS. } \\
\hline & & & & \multirow{2}{*}{$\begin{array}{c}\begin{array}{c}\text { Tiempo en otro } \\
\text { servicio }\end{array} \\
\% \\
\end{array}$} & \multirow{2}{*}{$\begin{array}{c}\text { Tiempo en servicio } \\
\text { CRED } \\
\% \\
\end{array}$} & \multirow{2}{*}{$\begin{array}{c}\begin{array}{c}\text { Tiempo de } \\
\text { espera, sin } \\
\text { recibir atención }\end{array} \\
\% \\
\end{array}$} \\
\hline & & & & & & \\
\hline CS & Lonya Grande & 3 & 49,3 & 2,7 & 22,3 & 75,0 \\
\hline HA & Santiago Apóstol & 5 & 217,2 & 1,4 & 8,1 & 90,5 \\
\hline CS & Haquira & 6 & 77,8 & 23,3 & 26,8 & 49,9 \\
\hline $\mathrm{H}$ & Tambobamba & 6 & 69,3 & 7,2 & 54,8 & 38,0 \\
\hline CS & Putacca & 6 & 54,0 & 23,5 & 53,1 & 23,5 \\
\hline CS & Vilcashuamán & 6 & 79,0 & 10,5 & 48,5 & 40,9 \\
\hline CS & San Marcos & 6 & 175,0 & 2,1 & 11,0 & 87,0 \\
\hline $\mathrm{H}$ & $\begin{array}{l}\text { San Javier } \\
\text { Bellavista }\end{array}$ & 3 & 128,0 & 0,3 & 11,7 & 88,0 \\
\hline CS & Paucartambo & 3 & 168,3 & 2,0 & 7,7 & 90,3 \\
\hline $\mathrm{H}$ & Santo Tomás & 3 & 79,7 & 11,7 & 19,7 & 68,6 \\
\hline CS & Huando & 3 & 92,0 & 1,8 & 35,1 & 63,0 \\
\hline CS & Paucarbamba & - & - & - & - & - \\
\hline CS & Puerto Inca & 4 & 49,8 & 9,5 & 36,7 & 53,8 \\
\hline PS & Margos & 2 & 72,0 & 0,0 & 52,1 & 47,9 \\
\hline CS & Isivilla & 2 & 150,5 & 21,3 & 21,9 & 56,8 \\
\hline CS & San Anton & 3 & 59,7 & 26,3 & 18,4 & 55,3 \\
\hline CS & Aguaytia & 3 & 136,7 & 13,9 & 25,1 & 61,0 \\
\hline \multirow[t]{2}{*}{ CS } & San Fernando & 3 & 166,7 & 4,6 & 5,0 & 90,4 \\
\hline & Total & 67 & 106,0 & 7,9 & 22,4 & 69,7 \\
\hline
\end{tabular}

EE. SS.: Establecimiento de Salud, CRED: Crecimiento y desarrollo, CS: centro de salud, HA: hospital de apoyo, H: hospital.

La demora en la atención es uno de los aspectos que más causó incomodidad en las madres participantes en los GF, y su percepción fue que ante esta situación hay desidia por parte del personal, sienten que no las respetan, ni les prestan atención (Tabla 4). Dicha demora fue confirmada en la OD de turnos completos de la atención de CRED (Tabla 6), encontrándose que más del $40 \%$ del tiempo del turno correspondió a demoras en la atención del usuario por salidas sin motivo claro del ambiente de atención, interrupciones por personal del mismo establecimiento de salud, y actividades personales de los responsables de la atención. Solo el $24,3 \%$ del tiempo correspondió a actividades generaban beneficio al usuario (agregaban valor).

Tabla 6. Distribución del tiempo de permanencia en el establecimiento de salud para la atención de CRED

\begin{tabular}{|c|c|c|c|c|c|c|c|}
\hline & & $\begin{array}{c}\text { Turnos } \\
\text { observados }\end{array}$ & Agrega valor & $\begin{array}{l}\text { Registro de } \\
\text { información }\end{array}$ & $\begin{array}{c}\text { Administrativo } \\
\text { obligatorio }\end{array}$ & Desplazamiento & $\begin{array}{l}\text { Demoras/ Personal } \\
\text { fuera del servicio }\end{array}$ \\
\hline & & $\mathbf{n}$ & $\%$ & $\%$ & $\%$ & $\%$ & $\%$ \\
\hline$\overline{\mathrm{CS}}$ & Lonya Grande & 1 & 45,5 & 32,5 & 15,2 & 2,5 & 4,4 \\
\hline $\mathrm{HA}$ & $\begin{array}{l}\text { Santiago } \\
\text { Apóstol }\end{array}$ & 1 & 24,1 & 35,6 & 0,0 & 0,0 & 40,4 \\
\hline CS & Haquira & 1 & 19,4 & 0,5 & 1,4 & 0,0 & 78,8 \\
\hline $\mathrm{H}$ & Tambobamba & 2 & 15,8 & 7,7 & 33,5 & 0,5 & 42,4 \\
\hline CS & Putacca & 1 & 46,1 & 8,4 & 18,4 & 0,0 & 27,2 \\
\hline CS & Vilcashuamán & 1 & 40,9 & 12,4 & 29,9 & 1,9 & 14,8 \\
\hline $\mathrm{CS}$ & San Marcos & 1 & 22,8 & 7,8 & 12,8 & 0,7 & 55,8 \\
\hline $\mathrm{H}$ & $\begin{array}{l}\text { San Javier } \\
\text { Bellavista }\end{array}$ & 2 & 24,9 & 30,2 & 5,6 & 0,3 & 39,0 \\
\hline CS & Paucartambo & 1 & 33,7 & 4,6 & 27,3 & 1,8 & 32,6 \\
\hline $\mathrm{H}$ & Santo Tomás & 2 & 19,3 & 0,9 & 19,0 & 2,9 & 57,9 \\
\hline CS & Huando & 3 & 8,3 & 35,6 & 0,5 & 0,5 & 55,2 \\
\hline CS & Paucarbamba & 1 & 19,2 & 9,7 & 20,7 & 1,5 & 48,9 \\
\hline CS & Puerto Inca & 1 & 6,5 & 0,7 & 73,1 & 0,0 & 19,6 \\
\hline PS & Margos & 1 & 27,3 & 17,9 & 29,9 & 0,2 & 24,7 \\
\hline CS & Isivilla & 1 & 0,0 & 0,0 & 6,9 & 1,4 & 91,7 \\
\hline CS & San Anton & 1 & 15,0 & 3,8 & 61,0 & 2,9 & 17,3 \\
\hline CS & Aguaytia & 1 & 37,0 & 3,8 & 43,2 & 0,0 & 15,9 \\
\hline \multirow[t]{2}{*}{ CS } & San Fernando & 1 & 51,2 & 7,6 & 0,0 & 1,8 & 39,4 \\
\hline & Total & 23 & 24,3 & 12,1 & 20,6 & 1,0 & 42,0 \\
\hline
\end{tabular}

CRED: Crecimiento y desarrollo, CS: centro de salud, HA: hospital de apoyo, H: hospital. 


\section{MENSAJES Y METODOLOGÍA USADA PARA PROPORCIONAR INFORMACIÓN DURANTE LA ATENCIÓN}

Las OD de las consultas de CRED evidenciaron que en la mayoría (18 de un total de 23 acompañamientos, 78,3\%) de las atenciones en menores de 6 meses, el personal de salud cumplió con indagar sobre la frecuencia de la lactancia materna y sobre la ingesta de otros líquidos distintos a ella. En cinco casos $(21,7 \%)$ faltaron preguntas apropiadas a las madres sobre posibles problemas durante la lactancia o derivados de la lactancia tales como dolor, dificultades para adoptar posición cómoda u observación de grietas o mastitis. Por otro lado, en los GF se identificó que los mensajes relacionados a la lactancia materna fueron los más recordados por las madres como parte de la consejería que brinda el servicio de CRED, principalmente: recomendaciones para asegurar una buena frecuencia de amamantamiento, e información sobre la importancia de la leche materna exclusiva hasta que el menor cumpla los 6 meses de edad. Además, las madres manifestaron que frecuentemente se les orientaba sobre la forma correcta e importancia del lavado de manos, con sesiones demostrativas en algunas oportunidades.

Con relación a la información sobre nutrición, la OD de 44 consultas CRED de niños de 6 a 36 meses evidenció que en cerca de dos tercios de las consultas se preguntó a la madre sobre la consistencia, cantidad, frecuencia y variedad de la dieta que recibe el niño. Sin embargo, en menos de la mitad de los casos se preguntó si el niño tiene plato propio y en solo $20 \%$ si se añadía una cucharadita de aceite a la comida del niño. En los GF, la mayoría de madres comentaron que los profesionales de la salud les daban diversas indicaciones para la alimentación adecuada de sus hijos, y que en numerosas oportunidades les entregaron suplementos de fierro y otros micronutrientes ("chispitas").

En la mayoría de los GF las participantes recordaron que los profesionales de salud les habían explicado que ante la aparición de fiebre, tos y cualquier otro síntoma o cambio mayor en el comportamiento del niño, tenían que acudir rápidamente al establecimiento de salud para que el menor sea revisado. Sin embargo, también hubo grupos focales mayormente en regiones de la sierra, donde las participantes comentaron que no se les dio ese tipo de información, y que desconocen sobre el tema.

Por otro lado, si bien la mayoría de madres mostraron interés de recibir mayor información para el cuidado de salud de los niños, también solicitaron que la información sea en su idioma (quechua en regiones de sierra). Refirieron además que la metodología demostrativa, dinámica y participativa para brindar la información facilita una mayor comprensión. Por su parte, los profesionales de salud confirmaron la importancia de contar con materiales educativos culturalmente adecuados a la realidad de las poblaciones con las que trabajan.

\section{DISCUSIÓN}

Mediante el uso de metodología cuantitativa y cualitativa, el presente estudio proporciona información sobre elementos importantes de la calidad de atención de niños menores de 3 años en servicios de CRED de nueve departamentos con índices altos de pobreza en Perú. A diferencia de otros trabajos que se limitan al análisis de la dimensión científica-técnica u objetiva de la atención infantil (15), el estudio añade el análisis de las percepciones sobre la atención desde las perspectivas de los usuarios (madres) y de los proveedores (profesionales de salud) de los servicios de CRED.

La triangulación de la información colectada evidenció que tanto las madres como los profesionales de salud coincidieron en manifestar que existen limitaciones de infraestructura, equipamiento, materiales y personal que afectarían la atención de CRED de calidad. Los profesionales son conscientes de que algunos de los ambientes, y el equipamiento requerido para la atención no se ajusta al mínimo establecido según la norma nacional ${ }^{(10)}$. Según la norma, el consultorio para la atención debe ser localizado preferentemente en la planta baja y de fácil acceso al público, con un espacio mínimo de $12 \mathrm{~m}^{2}$, con buena ventilación y libre de ruidos. También se especifica claramente requisitos mínimos para el mobiliario, los equipos (balanza pediátrica, balanza de pie, infantómetro y tallímetro), material médico y evaluación de desarrollo, material educativo y material de curación y aseo. En relación al personal de salud, la norma señala que cada establecimiento de salud de acuerdo a su categoría debe contar como mínimo con una enfermera o técnico capacitado, y de un equipo multidisciplinario para realizar un manejo integral de los problemas del CRED.

Estos mismos problemas encontrados en el estudio estarían presentes en la mayoría de los servicios del primer nivel de atención de acuerdo con una evaluación cuali-cuantitativa realizada por el MINSA en el 2010 en diez regiones del Perú. La evaluación concluye que el primer nivel de atención no estaría asumiendo el rol de ser la puerta de entrada al sistema de salud debido a su débil funcionamiento y pobre interrelación con los otros niveles de atención ${ }^{(16)}$. La limitada asignación presupuestal sería un obstáculo para que se cumplan los estándares de gestión, organización y prestación de servicios de salud, trayendo como consecuencia una débil capacidad de respuesta frente a las necesidades y demandas de la población. A esto se suma una débil capacidad de gestión local, para los procesos de planeamiento y conducción del establecimiento de salud, que en el caso de los EE. SS. del estudio, se traduce en 
una pobre organización del servicio en términos del flujo y secuencialidad de la atención.

Las coincidencias en percepciones entre madres y profesionales de salud fueron menores cuando el tema abordado fue el tiempo de espera para la atención. Mientras que para los profesionales la demora de la atención parece ser una situación habitual producto de una elevada demanda de atención que supera ampliamente los recursos humanos disponibles; para las madres, la demora es el aspecto que genera mayor incomodidad (insatisfacción) durante la atención. Dicha demora fue constatada durante la OD de los turnos de atención y consultas de CRED, y se deben principalmente a salidas del personal de salud del ambiente de atención sin motivo claro, interrupciones por personal del mismo establecimiento de salud, y actividades personales del personal de salud. Se ha descrito que con un menor tiempo de espera aumenta la percepción de satisfacción por parte de los pacientes ${ }^{(17,18)}$, y que un alto nivel de satisfacción promueve conductas positivas tales como conformidad, continuidad y longitudinalidad con los proveedores de salud ${ }^{(19)}$. La longitudinalidad es un atributo importante de la atención primaria ${ }^{(20)}$, considerado indispensable en servicios como los de CRED en donde se establecen relaciones personales a largo plazo entre profesionales de salud y madres. La longitudinalidad no solo favorece el abordaje de los problemas de los niños (diagnóstico oportuno y tratamiento apropiado), sino que también permite prevenir la aparición de enfermedades y/o problemas de crecimiento y desarrollo.

En muchos de los GF, se evidenció que las madres sabían muy bien que la mayor parte de la demora de la atención se debía a incumplimiento de horarios, de obligaciones, e indiferencia por parte del personal. Como usuarias del servicio de CRED no solo esperan que el personal que atiende a sus niños sea calificado y con experiencia, sino también que se destaque por su espíritu servicial y calidez en la atención. En diversos estudios en países de Latinoamérica ${ }^{(21,22)}$, también usuarios del primer nivel de atención coincidieron en señalar a los largos tiempos de espera, las omisiones en las acciones de revisión y diagnóstico, la complejidad de los trámites de consulta y el maltrato por parte del personal como los principales elementos que afectan la calidad de la atención. Existe consenso de que no se puede lograr una atención de calidad si la calidez no es parte ella ${ }^{(23,24)}$. La calidez o empatía, es el trato amable, la atención individualizada que se provee a los usuarios, el deseo de comprender sus necesidades con precisión y encontrar las respuesta más adecuadas a ellas ${ }^{(23)}$. El comportamiento empático favorecería la alianza preventiva-terapéutica posibilitando que la madre brinde más información sobre su niño, se muestre más comprometida con su cuidado y con mayor nivel de satisfacción, disminuyendo su ansiedad e incrementando la calidad de vida de cada miembro de la familia.

La calidad percibida por el usuario, entendida como la diferencia entre lo que el usuario espera del servicio y lo que dice haber obtenido, depende fundamentalmente de cómo se produce la interacción usuario-proveedor de salud y de las expectativas que previamente se haya formado de aquella interacción ${ }^{(25,26)}$. La comunicación apropiada (con empatía) en cada interacción incrementa significativamente la satisfacción del usuario ${ }^{(24)}$ y la probabilidad de obtener un resultado final satisfactorio de la prestación del servicio ${ }^{(27)}$. Por ello, la norma técnica nacional resalta la importancia de la consejería en los controles de CRED, como un proceso educativo comunicacional que se desarrolla de manera participativa entre el prestador de salud y los padres o responsables del cuidado del niño con el objetivo de ayudarlos a identificar los factores que influyen en su salud, nutrición y desarrollo, analizar los problemas encontrados en la evaluación e identificar acciones y prácticas que permitan mantener su crecimiento y desarrollo adecuado ${ }^{(10)}$. La triangulación de los hallazgos de la OD durante la consulta de CRED con los de los GF evidenció que la consejería viene cumpliendo con este objetivo en la mayoría de EE. SS. La consejería se focalizó en el mejoramiento de las prácticas de cuidado del niño, así como en el uso de los recursos disponibles en la familia, y permitió establecer un acuerdo negociado sobre las prácticas y acciones identificadas, fortaleciendo aquellas que son adecuadas y corrigiendo las de riesgo. Sin embargo, tres oportunidades de mejora fueron también identificadas: el desarrollo y entrenamiento de habilidades interpersonales y comunicacionales en los profesionales de salud, la mayor incorporación de metodologías demostrativas para reforzar los mensajes sobre prácticas adecuadas de cuidado infantil, y la necesidad de brindar consejería en la lengua materna de los padres.

Es necesario reconocer que los estudios con componente mixto (cuali-cuantitativo) podrían tener ciertas limitaciones, algunas relacionadas con la percepción de quien brinda la información (sea paciente, familiar o personal de salud), otras con la modificación de las características de la prestación cuando el personal de salud se percibe observado, o con la disponibilidad de ciertos recursos que se encuentran presentes durante el estudio, pero que podrían modificarse en el tiempo. El estudio, si bien no está exento de algunas de estas limitaciones, utiliza una selección aleatoria de establecimientos que minimiza la presencia de algunos de los sesgos (sobre todo cuantitativos), muestra un punto de referencia y evidencia dificultades que deben ser corregidas para la mejora de la atención del niño menor de 36 meses en los EE. SS. en un servicio crítico y directamente relacionado con su crecimiento y desarrollo.

Una de las funciones principales de los sistemas de salud es la provisión de servicios equitativos, oportunos, efectivos y seguros, en los que la calidad óptima de la atención es un componente clave para garantizarlos ${ }^{(28)}$. Por ello, 
los sistemas de salud deben incorporar el análisis permanente de la calidad de atención para identificar debilidades y áreas de oportunidad que permitan la reorientación de los servicios y una mejor asignación y uso de los recursos. El presente estudio encontró debilidades en la atención CRED (limitada infraestructura, equipamiento, y materiales; limitadas capacidades gerenciales y de organización a nivel local, escasos recursos humanos; etc.), que requieren mejor abordaje por el Estado Peruano como parte del componente de fortalecimiento del primer nivel de atención de la reforma del sector salud ${ }^{(16)}$. El componente promueve un nuevo modelo de atención integral en salud que centra su intervención en las necesidades de la persona, familia y comunidad, dando énfasis a las acciones de promoción de la salud y prevención de la enfermedad sin descuidar las actividades de recuperación y rehabilitación. Busca la reorientación de los servicios de salud hacia la calidad de atención, ya que el primer nivel de atención debe ser la puerta de entrada y el primer contacto de la población con el sistema de atención de salud.

Con base en la evidencia disponible, es crucial el fortalecimiento de los servicios de CRED en zonas rurales de los departamentos con los índices más altos de pobreza del Perú con la finalidad de reducir aun más la mortalidad y la desnutrición infantil. Tres actividades claves requieren ser priorizadas: la promoción de prácticas adecuadas y recursos de cuidados familiares y comunitarios a niños menores de 3 años (incluye capacitación del personal de salud y desarrollo de sus habilidades interpersonales y de comunicación, implementación de estrategias de educación y comunicación con enfoque intercultural, etc.), el mejoramiento de la capacidad resolutiva de las redes de servicios de salud con infraestructura y equipamiento, y el fortalecimiento de la capacidad de gobierno regional y local para el funcionamiento del sistema de salud.

Agradecimientos: la investigación contó con el apoyo del Programa de Apoyo a la Reforma del Sector Salud (PARSALUD II) del Ministerio de Salud, proyecto de inversión pública financiado con recursos del tesoro público y endeudamiento externo proveniente del Banco Interamericano de Desarrollo (BID) y el Banco Mundial (BM).

Contribuciones de autoría: LGCM, CMJ, JEVH participaron en la concepción y el diseño del estudio, LGCM, CMJ participaron en la recolección de datos. Todos participaron en el análisis e interpretación de los datos. JEVH, YRC y AR redactaron el artículo y todos los autores revisaron los borradores y la versión final, realizando importantes contribuciones.

Conflictos de interés: los autores declaran no tener conflictos de interés.

Fuente de financiamiento: la investigación contó con el apoyo del Programa de Apoyo a la Reforma del Sector Salud (PARSALUD II) del Ministerio de Salud, proyecto de inversión pública financiado con recursos del tesoro público y endeudamiento externo proveniente del Banco Interamericano de Desarrollo (BID) y el Banco Mundial (BM).

\section{REFERENCIAS BIBLIOGRÂFICAS}

1. Instituto Nacional de Estadística e Informática. Informe Técnico: Evolución de la Pobreza Monetaria 2007-2012. Lima: INEI; 2013.

2. Instituto Nacional de Estadística e Informática. Encuesta Demográfica y de Salud Familiar - ENDES 2013. Lima: INEI; 2014.

3. Black RE, Allen LH, Bhutta ZA, Caulfield LE, de Onis M, Ezzati $\mathrm{M}$, et al. Maternal and child undernutrition: global and regional exposures and health consequences. Lancet. 2008;371(9608):243-60. doi: 10.1016/S0140-6736(07)61690-0.

4. Black RE, Victora CG, Walker SP, Bhutta ZA, Christian P, de Onis M, et al. Maternal and child undernutrition and overweight in low-income and middle-income countries. Lancet. 2013;382(9890):427-51. doi: 10.1016/S0140-6736(13)60937-X.
5. Prado EL, Dewey KG. Nutrition and brain development in early life. Nutr Rev. 2014;72:267-84. doi:10.1111/ nure.12102

6. Victora CG, Adair L, Fall C, Hallal PC, Martorell R, Richter L, et al. Maternal and child undernutrition: consequences for adult health and human capital. Lancet. 2008;371(9609):340-57. doi: 10.1016/S0140-6736(07)61692-4.

7. Organización Panamericana de la Salud. Manual para la Vigilancia del Desarrollo Infantil en el Contexto de AIEPI. Washington D.C.: PAHO; 2011.

8. Hutchison T, Nicoll A. Developmental screening and surveillance. Br J Hosp Med. 1988;39:22-4, 28-9.

9. Walker SP, Wachs TD, GranthamMcGregor S, Black MM, Nelson CA, Huffman SL, et al. Inequality in early childhood: risk and protective factors for early child development. Lancet. 2011;378(9799):1325-38. doi: 10.1016/S0140-6736(11)60555-2.

10. Perú, Ministerio de Salud. Norma Técnica de salud para el control del Crecimiento y Desarrollo de la niña y el niño menor de cinco años. Lima: MINSA; 2011.

11. Donabedian A. The quality of care. How can it be assessed?. JAMA. 1988;260(12):1743-8.

12. Ministerio de Salud del Perú. Estudio de Factibilidad del Programa de Apoyo a la Reforma del Sector Salud. Lima: PARSALUD; 2008.

13. Instituto Nacional de Estadística e Informática. Censo Nacional de Población y Viviendas. Lima: INEI; 2007.

14. Patton MQ. Qualitative Research \& Evaluation Methods. Beverly Hills, CA: SAGE Publications; 2002. 
15. Furtado MC, Braz JC, Pina JC, de Mello DF, de Lima RA. Assessing the care of children under one year old in Primary Health Care. Rev Lat Am Enfermagem. 2013;21(2):554-61.

16. Perú, Ministerio de Salud. Plan Nacional de Fortalecimiento del Primer Nivel de Atención 2011-2021. Lima: MINSA; 2011.

17. Reyes H, Tomé P, Gutiérrez G, Rodríguez L, Orozco M, Guiscafré H. Mortality due to diarrhea in Mexico: a problem of accessibility or quality of care?. Salud Publica Mex. 1998;40(4):316-23.

18. Camacho F, Anderson R, Safrit A, Jones AS, Hoffmann P. The relationship between patient's perceived waiting time and officebased practice satisfaction. N C Med J. 2006;67(6):409-13.

19. Zastowny TR, Roghmann KJ, Cafferata GL. Patient satisfaction and the use of health services. Explorations in causality. Med Care. 1989;27(7):705-23.

20. Rosas Prieto AM, Narciso Zarate V, Cuba Fuentes MS. Atributos de la Atención Primaria de Salud (A.P.S): Una visión desde la Medicina Familiar. Acta Med Peruana. 2013;30(1):42-7.
21. Ortiz Espinosa RM, Muñoz Juárez $S$, Martin Del Campo D, Torres Carreño E. Consulta externa en instituciones de salud de Hidalgo, México, según la opinión de los usuarios. Rev Panam Salud Publica 2003;13(4):229-38. http://dx.doi.org/10.1590/S102049892003000300005.

22. Delgado-Gallego ME, Vázquez-Navarrete ML, de Moraes-Vanderlei L. [Quality of health-care according to social actors' frameworks of meaning in Colombia and Brazil]. Rev Salud Publica (Bogotá). 2010;12(4):533-45.

23. Leal Quevedo F. Calidad y calidez en la atención pediátrica. Arch Argent Pediatría. 2004;102(5):390-3.

24. Mesquita M, Pavlicich V, Benitez S. Percepción de la calidad de atención y accesibilidad de los consultorios pediátricos públicos en Asunción y Gran Asunción. Pediatría Asunción. 2008;35: 11-7.

25. Wonderlich S. Key Topics in Healthcare Management: Understanding the Big Picture. New York, NY: Radcliffe Publishing; 2007.

26. Thielscher C. How to Find the Right Approach to Quality Measurement:
Determinants of Quality and Its Measurement in Healthcare. World Med Health Policy. 2010;2:375-85. doi:10.2202/1948-4682.1036

27. García Millán Á. La información al paciente como pieza clave de la calidad asistencial. Rev Clin Med Fam. 2009;2(6):275-9. doi:10.4321/S1699695X2009000100005.

28. Thomson S, Osborn R, Squires D, Jun M. International Profiles of Health Care Systems, 2012 Australia, Canada, Denmark, England, France, Germany, Iceland, Italy, Japan, the Netherlands, New Zealand, Norway, Sweden, Switzerland, and the United States. New York, NY: The Commonwealth Fund; 2012.

Correspondencia: Yuleika Rodríguez Calviño Dirección: Calle Federico Villarreal 351.

Dpto 502-B. Miraflores. Lima

Teléfono: (511) 966361734

Correo electrónico:yrodriguez@parsalud.gob.pe

\section{Consulte la versión electrónica de la} Revista Peruana de Medicina Experimental y Salud Pública en

\section{www.pubmed.gov}

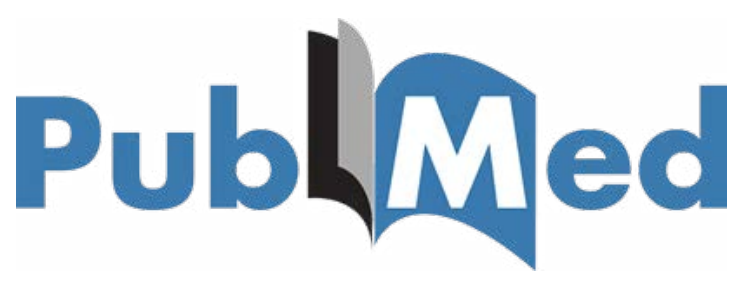

